\title{
Diets of tadpoles from a temporary pond in southeastern Brazil (Amphibia,Anura)
}

\author{
Denise de C. Rossa-Feres ${ }^{1}$, Jorge $\operatorname{Jim}^{2} \&$ Mariluce Gonçalves Fonseca ${ }^{3}$
}

${ }^{1}$ Corresponding author. Departamento de Zoologia e Botânica, Universidade Estadual Paulista. Rua Cristovão Colombo 2265, 15054-000 São José do Rio Preto, São Paulo,Brasil. E-mail: denise@dzb.ibilce.unesp.br

2 Departamento de Zoologia, Universidade Estadual Paulista. Distrito de Rubião Júnior, 18618-000 Botucatu, São Paulo, Brasil.

${ }_{3}^{3}$ Departamento de Ciências Biológicas, Faculdades FAFIBE. Rua Orlando França de Carvalho 325, 14071-070 Bebedouro, São Paulo, Brasil.

\begin{abstract}
The diet of tadpoles of 13 anuran species was determined to verify whether food resource partitioning occurs and whether the degree of diet similarity is related to taxonomic affinity. Tadpoles of all species studied were mainly herbivorous, except for these of Leptodactylus fuscus (Schneider, 1799) which were mycophagous. Although some species had exclusive items in their diet, most tadpole species ingested the same items, but differed in the amount of each item consumed. Two guilds were found: tadpoles that feed on diatoms on the pond bottom, and tadpoles that feed on Oedogonium Link, 1820 algae in midwater. Diet similarity was related to the taxonomic relationship, microhabitat and feeding behavior of tadpoles indicating that the community organization is complex and resulting from the interaction of several parameters.
\end{abstract}

KEY WORDS. Community ecology, food partitioning, microalgae.

RESUMO. Dieta dos girinos de um açude temporário no sudeste do Brasil (Amphibia, Anura). Determinou-se a dieta dos girinos de 13 espécies de anuros, com o objetivo de verificar se ocorre partilha de recursos alimentares e se o grau de similaridade na dieta é relacionado ao parentesco, inferido pela proximidade taxonômica. Os girinos de todas as espécies estudadas foram preponderantemente herbívoros, exceto os de Leptodactylus fuscus (Schneider, 1799) que foram micófagos. Embora algumas espécies tenham apresentado itens exclusivos em sua dieta, os girinos da maioria das espécies ingeriram os mesmos itens, diferindo na quantidade consumida de cada item. Duas guildas foram encontradas: girinos que se alimentam de diatomáceas no fundo do corpo d'água e girinos que se alimentam de algas do gênero Oedogonium Link, 1820 à meia-água. A similaridade na dieta foi relacionada à proximidade taxonômica, ao microhabitat e ao comportamento de alimentação, indicando que a organização desta comunidade é complexa e resultante da interação entre vários fatores.

PALAVRAS CHAVE. Ecologia de comunidade, partilha de alimento, microalgas, sudeste do Brasil.

Most tadpoles are primarily herbivorous (Alford 1999) consuming a wide variety of algal taxa as well as detritus, viruses, bacteria, protists, plant fragments, pollen grains, fungi, various kinds of small animals, anuran eggs, and other tadpoles (KupFerberg et al. 1994, Hoff et al. 1999).

Field studies of tadpole diets, including systematic and comparative evaluation of the food habits of tadpoles are rare (Alford 1999, Hoff et al. 1999) and few studies have detailed how food differences might influence community organization (Díaz-Paniagua 1985, Inger 1986, Lajmanovich 2000). As a consequence, little consensus exists regarding the occurrence of food partitioning and its role in the organization of tadpole communities. Belova (1964) observed that the diet of Rana ridibunda Pallas, 1771 (Ranidae) tadpoles collected from different bodies of water was similar. Heyer (1973), Johnson (1991), SEAle \& BeCKVAR (1980) and Lajmanovich (1997) showed that tadpoles feed on what is available in their habitat. Heyer (1976) and Lajmanovich (2000) concluded that food plays no important role in habitat partitioning among species, whereas INGER (1986) observed that partition food was intertwined with distribution in space, and WASSERSUG (1980) suggested that differences in the internal oral characteristics may be related to preferences to different sizes of food particles in the microhabitats of tadpoles.

In the present study, we determined the diet of tadpoles of 13 anuran species in a temporary pond, southeastern Brazil. The patterns of breadth and overlap in food resource use were determined to analyze whether partitioning occurs and whether the degree of diet similarity is related to taxonomic affinity. 


\section{MATERIAL AND METHODS}

\section{Study Site}

This study was conducted in an artificial, temporary pond in a pasture in Nova Itapirema, District of Nova Aliança (2111'S, 4942'W), State of São Paulo, Brazil. The pond, with clayey bottom and irregular shape, measured about $54 \times 22 \mathrm{~m}$ and was $0.7 \mathrm{~m}$ deep during the period of largest water volume. Water persisted for 5-7 months/year. The margins were covered mainly with grasses (Poaceae and Cyperaceae) and the aquatic vegetation consisted of Nympheaceae, Poaceae, Cyperaceae and Polygonaceae. The climate of the region is tropical, with an annual rainfall ranging from $1,100-1,250 \mathrm{~mm}$, $85 \%$ of which occurred during the rainy season from October to March (ARID \& BARCHA 1973).

\section{A nalysis of gut contents of tadpoles}

The tadpoles were collected at least once a month from October 1989 to March 1995, between 15:00 and 19:00 h, with a fine-mesh dipnet $\left(3 \mathrm{~mm}^{2}\right)$ and preserved immediately in $10 \%$ formalin. After identification, the animals were stored in $5 \%$ formalin and deposited in the amphibian collection of the Departamento de Zoologia e Botânica, Universidade Estadual Paulista (UNESP), Campus from São José do Rio Preto (DZSJ RP 1 to 128). Adults of these species were also deposited in same collection.

In a previous analysis was observed that the manicotto glandulare of tadpoles was usually empty and that food content did not vary in quantity or composition along the intestine. Thus, we just analyzed the content of the first centimeter of the intestine of five individuals of each species, in developmental stages 35-38 of Gosner (1960). This part of the gut was removed and its content was homogenized in $160 \mu$ of Transeau solution (BıCUDo \& BıCUdo 1970). Four semipermanent slides were mounted for each tadpole, with $40 \mu$ of this solution. In two slides, the food items were identified and counted in the entire area covered by the coverslip $\left(324 \mathrm{~mm}^{2}\right)$ at $200 x$ magnification. In the other two slides, the size of the food items was measured with a grid calibrated for the objective of the microscope at $160 x$ magnification. The algae were identified to genus according to BICUDO \& BICUDO (1970) and their microhabitat was defined on the basis of their morphology. Those with any attachment structures were considered periphytic and the remaining were planktonic. The position of the tadpoles in the water column was determined by direct observation, and derived from microhabitat of the ingested algae, and the tadpoles were considered benthic or nektonic midwater.

Diet diversity was calculated for each species by the Shannon-Wiener index $\left(\mathrm{H}^{\prime}\right)$ and the breadth of the niche for the types of food items ingested was calculated by Levins' measure (B) (KREBS 1999). The niche overlap was cal culated by the similarity index of Morisita-Horn $\left(\mathrm{C}_{H}\right)$ for the type (frequency of ingestion of each algal genus) and size (frequency of ingestion of each size category of algae) gradient of the food items. Over- lap values were considered high when surpassing $C_{H}=0.50$. Cluster analysis was applied to the similarity matrix obtained by the unweighted mean method (KREBS 1999).

\section{Species}

We analyzed the gut content of tadpoles of the following 13 species: Bufo schneideri Werner, 1894 (Bufonidae); Elachistocleis ovalis (Schneider, 1799) (Microhylidae); Leptodactylus fuscus (Schneider, 1799), L. podicipinus (Cope, 1862), Physalaemus centralis Bokermann, 1962, P. cuvieri Fitzinger, 1826, P. fuscomaculatus (Steindachner, 1864), P. nattereri (Steindachner, 1863) (Leptodactylidae); Hyla nana Boulenger, 1889, Phrynohyas venulosa (Laurenti, 1768), S. fuscomarginatus (A. Lutz, 1925), Scinax fuscovarius (A. Lutz, 1925), and S. similis (Cochran, 1952) (Hylidae). Reproductive adults of both Hyla nana Boulenger, 1889 and H. sanborni Schmidt, 1944 were found, but because tadpoles of these species could not be distinguished, they were lumped together as Hyla nana. Scinax fuscomarginatus tadpoles were rare and the diet of only one individual is described here. Six species form pairs of taxonomically related species (Physalaemus cuvieri and P. centralis; P. nattereri and P. fuscomaculatus; Scinax fuscovarius and S. similis).

During a five-year study, the tadpoles of these 13 species coexisted during some period in each year. Since the pool is temporary and accumulates only rain water, it is assumed that resource availability changed yearly within a narrow breadth. Thus, to characterize the diet of tadpoles, the gut contents were analyzed in individuals collected during their modal period of occurrence and in the years when they were most abundant (Tab. I). Besides, to minimize the risk of confounding differences between days (INGER 1986), gut contents of pairs of taxonomically related tadpoles were analyzed from specimens collected on the same day.

Table I. Dates of tadpole collection from a temporary pond in Nova Itapirema, São Paulo, Brazil.

\begin{tabular}{lc}
\hline \multicolumn{1}{c}{ Species } & Date \\
\hline Hyla nana & 25 Jan 90 \\
Physalaemus centralis & 7 March 90 \\
Physalaemus cuvieri & 7 March 90 \\
Scinax fuscomarginatus & 7 March 90 \\
Scinax fuscovarius & $20 \operatorname{Dec} 90$ \\
Scinax similis & $20 \operatorname{Dec} 90$ \\
Bufo schneider & $20 \operatorname{Dec} 90$ \\
Physalaemus nattereri & 29 Nov 94 \\
Physalaemus fuscomaculatus & 29 Nov 94 \\
Leptodactylus fuscus & 29 Nov 94 \\
Leptodactylus podicipinus & 29 Nov 94 \\
Elachistocleis ovalis & 29 Nov 94 \\
Phrynohyas venulosa & 8 Nov 94 \\
\hline
\end{tabular}




\section{RESULTS}

\section{Spectrum of food items}

The tadpole diets consisted mostly of microalgae, although fragments of higher plants (mainly leaves of Poaceae and Cyperaceae), fungal hyphae (mainly Deuteromycotina), Sarcomastigophora, Ciliophora, Rotifera, Nematoda, Tardigrada, and Crustacea were also found (Tab. II). Microalgae belonging to 44 genera were identified, diatoms (Bacillariophyceae) represented $8-54 \%$ of the diet of tadpoles of 13 species recorded in the temporary pond (Tabs III and IV).

Three items were found in the diet of all species: diatoms (Bacillariophyceae), Oedogonium (Oedogoniaceae) and Trachelomonas (Euglenaceae). On the other hand, fifteeen items were rare and found in the intestines of only one species (Tabs III and IV). The same occurred with other four items, that were found in a notable amount (> 5\%): Lyngbya Agardh, 1824 (Oscillatoriaceae) in Physalaemus cuvieri; Sorastrum Kutzing, 1845 (Hydrodictyaceae), Scenedesmus Meyen, 1829 (Scenedesmaceae) and Lepocinclis Perty, 1849 (Euglenaceae) in Bufo schneideri (Tabs III and IV).

\section{Diet diversity and similarity}

Tadpoles of seven species (Physalaemus centralis, P. cuvieri, P. fuscomaculatus, P. nattereri, Leptodactylus fuscus, L. podicipinus, and Bufo schneideri) occurred on the pond bottom, and tadpoles of Scinax fuscovarius, S. similis and Phrynohyas venulosa occurred in the midwater. Except for Bufo schneideri and Phrynohyas venulosa tadpoles, which ingested large amounts of planktonic algae, the remaining tadpoles mainly ingested periphytic algae (Fig. 1).

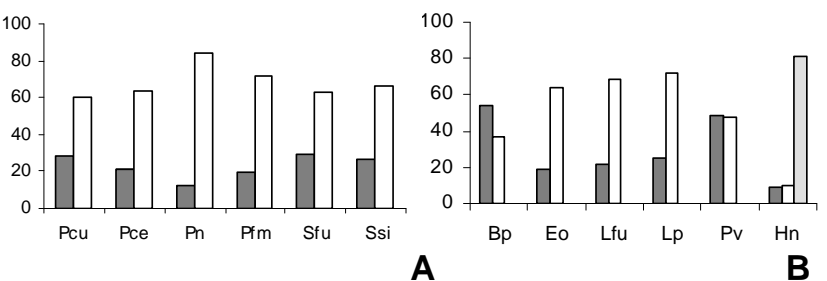

$\square$ Planktonic algae $\square$ Periphytic algae $\square$ Unidentified items

Figure 1. Percentage of food items of planktonic and periphytic habits in the diet of tadpoles of the three pairs of taxonomically related species (A) and of the other six species (B) in the temporary pond of Nova Itapirema, São Paulo.

The members of each species pair - Physalaemus cuvieri and P. centralis, P. nattereri and P. fuscomaculatus, Scinax fuscovarius and S. similis - were similar in diet composition (Fig. 2), with only 4-6 items not shared (Tab. III). Among Leptodactylus fuscus and $L$. podicipinus, congeneric species, but belonging to different species groups, the diet differed at least in terms of the most abundant item (Tab. IV). For the remaining species, there were 8-10 items not shared (Tab. IV). However, some of these species had diets similar to species belonging to the three taxonomically related pairs (e.g. Leptodactylus podicipinus with Physalaemus centralis; Elachistocleis ovalis with P. nattereri; Tabs III and IV). Similarity analysis demonstrated two major species clusters with overlap values higher than 0.70 (Fig. 3): (1) seven species which ingested mainly diatoms (Bacillariophyceae) and (2) two taxonomically related species (Scinax fuscovarius and S. similis) which ingested mainly algae of the genus Oedogonium. Three species with different diets do not

Table II. Categories of food items ingested and their percent frequencies (\%) in the diet of tadpoles of 13 species. (Bs) Bufo schneider, (Eo) Elachistocleis ovalis, (Hns) Hyla nana, (Lfu) Leptodactylus fuscus, (Lp) L. podicipinus, (Pce) Physalaemus centralis, (Pcu) P. cuvieri, (Pfm) P. fuscomaculatus, (Pn) P. nattereri, (Pv) Phrynohyas venulosa, (Sfu) S. fuscovarius, (Ssi) S. similis, (Sfm) Scinax fuscomarginatus. Except for S. fuscomarginatus (with only one specimen examined), five specimens of each species were examined. (UNI) Unidentified item.

\begin{tabular}{lccccccccccccc}
\hline \multicolumn{1}{c}{ Food items } & Bs & Eo & Hn & Lfu & Lp & Pce & Pcu & Pfm & Pn & Pv & Sfu & Ssi & Sfm \\
\hline Microalgae & 90.05 & 72.03 & 17.87 & 33.35 & 79.32 & 94.21 & 95.04 & 77.35 & 81.84 & 69.87 & 59.64 & 84.54 & 95.39 \\
Fungus & 0.72 & 1.10 & 0.28 & 34.60 & 3.60 & 1.14 & 1.20 & 8.97 & 4.99 & 2.94 & 0.61 & 0.85 & - \\
Fragments of angiosperms & 3.48 & 2.06 & 0.37 & 13.35 & 9.06 & 0.33 & - & 4.09 & 7.74 & 5.87 & 5.60 & 7.00 & 0.28 \\
Sarcomastigophora & 0.74 & 6.67 & 0.27 & 4.66 & 5.38 & 2.33 & 3.00 & 0.98 & 1.11 & 5.38 & 2.75 & 1.43 & 0.14 \\
Ciliophora & 0.09 & 0.11 & 0.02 & 1.97 & 0.21 & - & - & 0.17 & 0.09 & 2.07 & - & 1.83 & - \\
Rotifera & 2.90 & 5.23 & 0.57 & 5.05 & 1.62 & 0.11 & 0.02 & 2.89 & 2.01 & 13.07 & 1.00 & 3.35 & 0.55 \\
Nematoda & 0.17 & 0.22 & - & 1.15 & 0.26 & 1.18 & 0.34 & 0.52 & 0.24 & - & 1.00 & 0.20 & 0.14 \\
Tardigrada & 0.08 & - & - & 0.70 & 0.06 & - & - & - & 0.71 & 0.05 & 0.06 & 0.05 & - \\
Acari & - & - & - & 0.14 & - & - & - & 0.05 & 0.03 & - & - & - & - \\
Nematocera, larvae & - & - & - & - & - & - & 0.03 & - & - & - & - & - & - \\
Crustacea & 0.36 & 7.98 & 0.14 & 3.40 & 0.44 & 0.67 & 0.52 & 2.72 & 1.63 & 0.28 & 1.00 & 1.53 & 0.48 \\
UNI 1 & - & 4.64 & 72.54 & - & - & - & - & - & - & - & - & - & - \\
UNI 2 & - & - & 8.22 & - & - & - & - & - & - & - & - & - & - \\
\hline
\end{tabular}



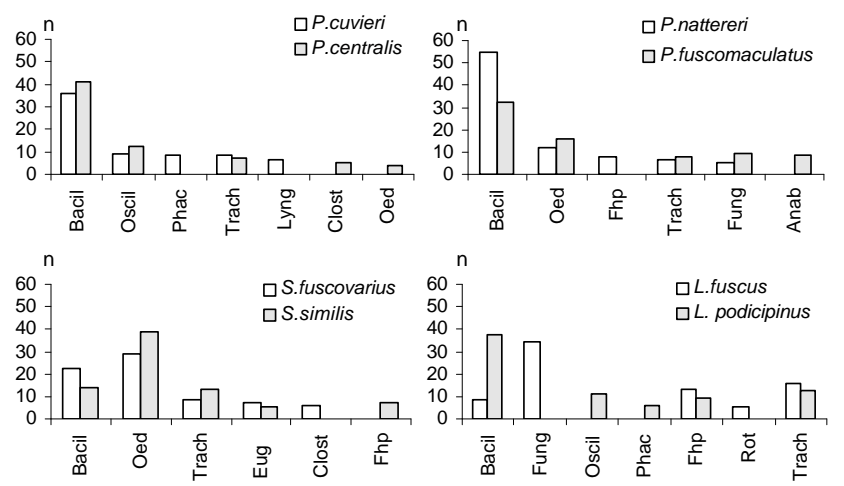

Figure 2. Five most abundant food items of each species of taxonomically related pairs and of the congenerics Leptodactylus fuscus and L. podicipinus. (Anab) Anabaena, (Bacil) Bacillariophyceae, (Clost) Closterium, (Eug) Euglena, (Fhp) fragment of a higher plant, (Fung) Fungal hyphae, (Lyng) Lyngbya, (Oed) Oedogonium, (Oscil) Oscillatoria, (Phac) Phacus, (Rot) Rotifera, (Trach) Trachelomonas.

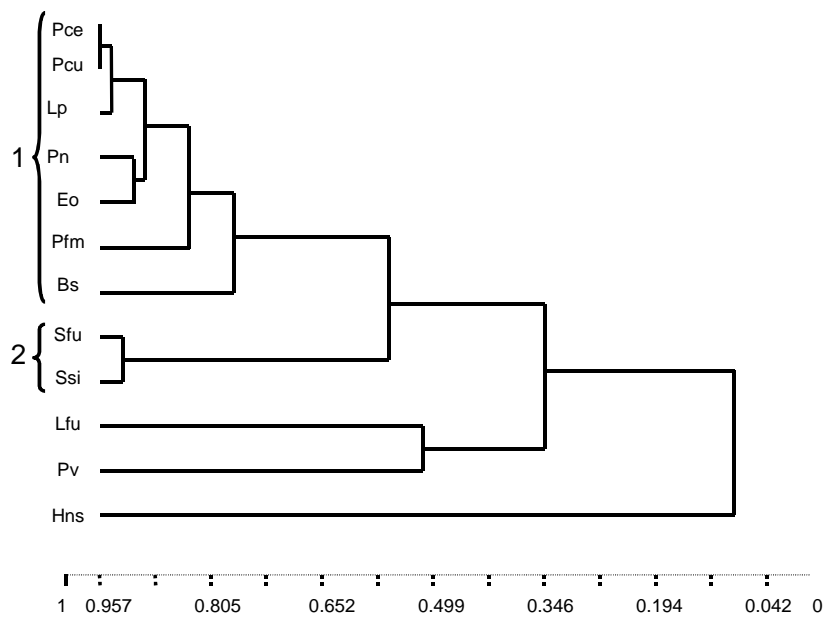

Figure 3. Clusters of species, indicating those with similarity higher than $\mathrm{C}_{\mathrm{H}}=0.70$ in the composition of the diet. For abbreviations see table II.

Table III. Abundance (mean \pm standard deviation, $n=5$ ) of the food items found in the gut content of tadpoles of the three pairs of taxonomically related species in the temporary pond of Nova Itapirema, São Paulo. The most abundant items for each species are in bold; (FA) fragment of angiosperms; (UNI) unidentified item.

\begin{tabular}{|c|c|c|c|c|c|c|}
\hline Food items & P. cuvieri & P. centralis & P. nattereri & P. fuscomaculatus & S. fuscovarius & S. similis \\
\hline \multicolumn{7}{|l|}{ Cyanophyta } \\
\hline Anabaena & $5.70 \pm 8.06$ & - & - & $8.76 \pm 9.77$ & $3.86 \pm 8.63$ & - \\
\hline Chroococcus & $0.23 \pm 0.38$ & $0.07 \pm 0.16$ & $0.06 \pm 0.09$ & $0.05 \pm 0.10$ & $0.23 \pm 0.21$ & $0.50 \pm 0.64$ \\
\hline Lyngbya & $6.25 \pm 8.60$ & $0.88 \pm 0.85$ & - & - & $0.25 \pm 0.30$ & $0.15 \pm 0.34$ \\
\hline Oscillatoria & $9.40 \pm 5.71$ & $12.34 \pm 13.55$ & $1.19 \pm 0.58$ & $5.56 \pm 3.67$ & $3.25 \pm 1.76$ & $3.40 \pm 3.00$ \\
\hline Spirulina & $0.10 \pm 0.12$ & - & - & - & $0.04 \pm 0.10$ & - \\
\hline Cyanophyceae (UNI) & $0.10 \pm 0.15$ & $0.11 \pm 0.12$ & - & - & - & - \\
\hline \multicolumn{7}{|l|}{ Chlorophyta } \\
\hline Actinotaenium & $0.25 \pm 0.17$ & $1.02 \pm 1.15$ & - & - & - & - \\
\hline Ankistrodesmus & - & $0.04 \pm 0.05$ & $0.49 \pm 0.16$ & $1.02 \pm 0.73$ & - & - \\
\hline Cladophora & - & - & $0.72 \pm 1.61$ & - & - & - \\
\hline Chlorococcum & $1.70 \pm 1.10$ & $1.28 \pm 0.90$ & - & - & - & - \\
\hline Closterium & $2.90 \pm 1.70$ & $5.02 \pm 2.89$ & $4.41 \pm 1.94$ & $2.86 \pm 1.58$ & $6.0 \pm 3.50$ & $2.70 \pm 1.20$ \\
\hline Coelastrum & - & - & $0.16 \pm 0.23$ & $0.54 \pm 0.56$ & $0.35 \pm 0.80$ & - \\
\hline Cosmarium & $1.35 \pm 1.85$ & $3.57 \pm 1.07$ & - & $0.25 \pm 0.35$ & $3.00 \pm 2.56$ & $3.61 \pm 2.81$ \\
\hline Desmidium & $0.74 \pm 0.87$ & $2.18 \pm 2.32$ & - & - & - & - \\
\hline Euastrum & $0.26 \pm 0.13$ & $0.67 \pm 0.61$ & - & - & $1.07 \pm 1.16$ & $0.32 \pm 0.43$ \\
\hline Gonatozygon & $0.34 \pm 0.47$ & $1.25 \pm 2.42$ & - & - & - & - \\
\hline Mesotaenium & - & $0.09 \pm 0.20$ & - & - & - & - \\
\hline Micrasterias & $0.40 \pm 0.36$ & $0.51 \pm 1.03$ & - & - & - & - \\
\hline Oedogonium & $3.00 \pm 2.30$ & $3.78 \pm 1.28$ & $11.86 \pm 7.26$ & $15.53 \pm 14.33$ & $29.00 \pm 24.24$ & $38.80 \pm 22.0$ \\
\hline Oocystis & $0.76 \pm 0.51$ & $0.89 \pm 0.76$ & $0.23 \pm 0.17$ & $0.63 \pm 0.73$ & $0.50 \pm 0.72$ & $1.00 \pm 0.85$ \\
\hline Pediastrum & - & $0.02 \pm 0.05$ & - & - & - & - \\
\hline Pleurotaenium & - & $0.04 \pm 0.10$ & - & - & $0.10 \pm 0.20$ & $0.12 \pm 0.27$ \\
\hline
\end{tabular}


Table III. Continued.

\begin{tabular}{|c|c|c|c|c|c|c|}
\hline Food items & P. cuvieri & P. centralis & P. nattereri & P. fuscomaculatus & S. fuscovarius & S. similis \\
\hline \multicolumn{7}{|l|}{ Chlorophyta (cont.) } \\
\hline Scenedesmus & $0.30 \pm 0.10$ & $0.57 \pm 0.29$ & - & $0.08 \pm 0.11$ & $0.61 \pm 0.40$ & $0.15 \pm 0.34$ \\
\hline Selenastrum & $0.03 \pm 0.06$ & $0.02 \pm 0.05$ & - & - & - & - \\
\hline Sorastrum & $0.03 \pm 0.06$ & $0.15 \pm 0.20$ & $0.05 \pm 0.12$ & $0.08 \pm 0.11$ & - & - \\
\hline Sphaerocystis & - & $0.08 \pm 0.08$ & $0.55 \pm 0.36$ & $0.70 \pm 0.78$ & $1.10 \pm 1.73$ & $0.70 \pm 0.62$ \\
\hline Spirogyra & $2.84 \pm 2.05$ & $2.58 \pm 1.11$ & - & - & - & - \\
\hline Staurastrum & $0.80 \pm 0.14$ & $0.86 \pm 0.33$ & $0.08 \pm 0.11$ & $0.09 \pm 0.13$ & - & $0.16 \pm 0.24$ \\
\hline Staurodesmus & $0.07 \pm 0.12$ & $0.02 \pm 0.05$ & - & - & - & - \\
\hline Xantidium & - & $0.02 \pm 0.05$ & - & - & - & - \\
\hline Chlorophyceae (UNI) & $0.33 \pm 0.46$ & - & - & - & - & - \\
\hline Bacillariophyta & $36.05 \pm 13.70$ & $40.83 \pm 7.98$ & $54.45 \pm 10.67$ & $32.29 \pm 8.91$ & $22.21 \pm 11.66$ & $13.63 \pm 8.00$ \\
\hline \multicolumn{7}{|l|}{ Euglenophyta } \\
\hline Eudorina & $0.51 \pm 0.61$ & $1.19 \pm 0.91$ & $0.03 \pm 0.08$ & - & - & - \\
\hline Euglena & $2.60 \pm 1.33$ & $3.25 \pm 2.77$ & $0.93 \pm 0.60$ & $1.34 \pm 0.42$ & $7.0 \pm 2.00$ & $5.45 \pm 2.23$ \\
\hline Lepocinclis & $0.66 \pm 0.40$ & $1.54 \pm 0.65$ & $0.03 \pm 0.08$ & $0.14 \pm 0.21$ & $0.21 \pm 0.40$ & - \\
\hline Phacus & $8.77 \pm 9.25$ & $2.25 \pm 0.95$ & $0.33 \pm 0.12$ & $0.56 \pm 0.33$ & $1.35 \pm 0.70$ & $0.70 \pm 1.30$ \\
\hline Strombomonas & $0.03 \pm 0.06$ & $0.08 \pm 0.11$ & $0.03 \pm 0.07$ & - & - & - \\
\hline Trachelomonas & $8.52 \pm 2.61$ & $7.18 \pm 3.23$ & $6.84 \pm 5.98$ & $7.87 \pm 5.19$ & $8.34 \pm 10.43$ & $13.0 \pm 16.28$ \\
\hline \multicolumn{7}{|l|}{ Chrysophyta } \\
\hline Dinobryon & - & - & - & - & $0.17 \pm 0.40$ & $0.15 \pm 0.34$ \\
\hline \multicolumn{7}{|l|}{ Dinophyta } \\
\hline Goniaulax & $0.02 \pm 0.04$ & $0.03 \pm 0.06$ & - & - & - & - \\
\hline Fungus & $1.20 \pm 0.87$ & $1.14 \pm 0.24$ & $4.99 \pm 2.88$ & $8.97 \pm 11.51$ & $0.61 \pm 0.51$ & $0.85 \pm 0.67$ \\
\hline FA & - & $0.33 \pm 0.41$ & $7.74 \pm 5.53$ & $4.09 \pm 1.70$ & $5.60 \pm 6.40$ & $7.00 \pm 10.00$ \\
\hline Thecamaeba & $3.0 \pm 1.36$ & $2.33 \pm 1.60$ & $1.11 \pm 1.04$ & $2.83 \pm 2.79$ & $2.75 \pm 2.13$ & $1.43 \pm 1.04$ \\
\hline Ciliophora & - & - & $0.09 \pm 0.20$ & $0.17 \pm 0.22$ & - & $1.83 \pm 3.00$ \\
\hline Rotifera & $0.02 \pm 0.04$ & $0.11 \pm 0.16$ & $2.01 \pm 1.31$ & $2.89 \pm 1.54$ & $1.00 \pm 0.64$ & $3.35 \pm 3.43$ \\
\hline Nematoda & $0.34 \pm 0.44$ & $1.18 \pm 1.97$ & $0.24 \pm 0.25$ & $0.52 \pm 0.79$ & $1.00 \pm 2.00$ & $0.20 \pm 0.33$ \\
\hline Tardigrada & - & - & $0.71 \pm 1.16$ & $0.98 \pm 0.96$ & $0.06 \pm 0.14$ & $0.05 \pm 0.11$ \\
\hline Acari & - & - & $0.03 \pm 0.07$ & $0.05 \pm 0.10$ & - & - \\
\hline Diptera (larvae) & $0.03 \pm 0.06$ & - & - & - & - & - \\
\hline Copepoda & - & $0.05 \pm 0.11$ & - & - & - & - \\
\hline Ostracoda & $0.12 \pm 0.20$ & $0.12 \pm 0.13$ & - & - & - & - \\
\hline Crustacea (UNI) & $0.40 \pm 0.17$ & $0.50 \pm 0.35$ & $0.44 \pm 0.35$ & $2.72 \pm 2.87$ & $1.00 \pm 0.32$ & $1.53 \pm 1.20$ \\
\hline UNI 3 & - & - & $0.12 \pm 0.27$ & - & - & - \\
\hline
\end{tabular}

belong to either cluster: Hyla nana, Phrynohyas venulosa and Leptodactylus fuscus.

The diversity and niche breadth of the food type ingested were high $\left(H^{\prime}>0.95\right.$ and $B>4.0$, respectively) for most of the species (Tab. V). Niche overlap was high $\left(C_{H}>0.60\right)$ for $42.4 \%$ of the 66 possible combinations of species pairs (Tab. VI). Hyla nana tadpoles, which mainly ingested an unidentified item resembling in shape to members of Bacillariophyceae, Leptodactylus fuscus tadpoles, which were preponderantly mycophagous, and Phrynohyas venulosa tadpoles, which mainly consumed planktonic algae, were the only ones presenting low niche overlap with the remaining species. The highest overlap occurred between Physalaemus centralis and P. cuvieri (Tab. VI).

The tadpoles ingested items ranging in size from 10- 
Table IV. Abundance (mean \pm standard deviation, $n=5$ ) of the food items found in the gut content of tadpoles of seven species in the temporary pond of Nova Itapirema, São Paulo. The most abundant items for each species are in bold; $(*)$ description of the diet of one individual; (FA) fragment of angiosperms; (UNI) unidentified item.

\begin{tabular}{|c|c|c|c|c|c|c|c|}
\hline Food items & L. fuscus & L. podicipinus & B. schneider & P. venulosa & E. ovalis & Hyla nana & *S. fuscomarginatus \\
\hline \multicolumn{8}{|l|}{ Cyanophyta } \\
\hline Anabaena & $0.18 \pm 0.42$ & $0.22 \pm 0.36$ & - & - & $0.64 \pm 0.77$ & - & 0.07 \\
\hline Chroococcus & - & $0.08 \pm 0.19$ & - & - & - & - & - \\
\hline Oscillatoria & $1.13+1.56$ & $10.89 \pm 8.33$ & $0.59 \pm 0.69$ & $0.05 \pm 0.09$ & $2.05 \pm 0.83$ & - & 0.07 \\
\hline Spirulina & - & - & - & - & $0.40 \pm 0.50$ & - & - \\
\hline \multicolumn{8}{|l|}{ Chlorophyta } \\
\hline Ankistrodesmus & $1.09 \pm 1.95$ & $0.52 \pm 0.27$ & $0.84 \pm 0.80$ & $0.06 \pm 0.11$ & - & $0.06 \pm 0.08$ & 0.14 \\
\hline Chaetophora & - & $0.23 \pm 0.36$ & - & - & - & - & - \\
\hline Closterium & $2.29 \pm 1.41$ & $4.30 \pm 1.11$ & $1.05 \pm 1.74$ & $2.04 \pm 0.47$ & $8.45 \pm 1.51$ & $1.10 \pm 1.07$ & 6.40 \\
\hline Coelastrum & $0.37 \pm 0.84$ & $0.20 \pm 0.19$ & $1.17 \pm 1.11$ & $0.34 \pm 0.43$ & - & - & 39.19 \\
\hline Cosmarium & $0.13 \pm 0.28$ & $0.18 \pm 0.24$ & $0.95 \pm 0.47$ & - & $0.04 \pm 0.08$ & $0.22 \pm 0.07$ & 7.10 \\
\hline Cruciginea & - & - & $1.37 \pm 0.66$ & - & - & - & 0.14 \\
\hline Desmidium & - & - & - & - & - & - & 0.21 \\
\hline Dictyosphaerium & - & - & $0.25 \pm 0.34$ & - & $0.06 \pm 0.12$ & - & 4.30 \\
\hline Euastrum & - & - & $0.65 \pm 0.43$ & $0.06 \pm 0.11$ & - & $0.11 \pm 0.15$ & 1.00 \\
\hline Gonatozygon & - & - & - & - & - & - & 7.83 \\
\hline Mougeotia & - & - & - & - & - & - & 0.14 \\
\hline Oedogonium & $1.94 \pm 1.93$ & $3.77 \pm 2.84$ & $3.43 \pm 3.23$ & $12.57 \pm 6.30$ & $2.30 \pm 1.05$ & $0.40 \pm 0.22$ & 2.60 \\
\hline Oocystis & $0.13 \pm 0.29$ & $0.20 \pm 0.28$ & $0.16 \pm 0.15$ & $0.30 \pm 0.29$ & $0.64 \pm 0.50$ & $0.04 \pm 0.08$ & 0.35 \\
\hline Scenedesmus & - & - & $6.23 \pm 3.14$ & $0.06 \pm 0.11$ & $0.06 \pm 0.13$ & - & 0.14 \\
\hline Sorastrum & - & $0.06 \pm 0.14$ & $7.21 \pm 2.37$ & - & $0.06 \pm 0.13$ & - & - \\
\hline Sphaerocystis & $0.12 \pm 0.28$ & $0.36 \pm 0.30$ & $0.10 \pm 0.14$ & $0.05 \pm 0.09$ & - & - & - \\
\hline Staurodesmus & - & - & $0.15 \pm 0.34$ & - & - & - & 0.14 \\
\hline Bacillariophyta & $8.81 \pm 5.06$ & $37.46 \pm 10.27$ & $27.56 \pm 7.0$ & $8.24 \pm 0.53$ & $45.57 \pm .40$ & $8.17 \pm 4.20$ & 3.20 \\
\hline \multicolumn{8}{|l|}{ Euglenophyta } \\
\hline Eudorina & $0.73 \pm 0.70$ & $0.20 \pm 0.19$ & - & $0.37 \pm 0.39$ & $0.18 \pm 0.17$ & - & 5.00 \\
\hline Euglena & $1.61 \pm 0.36$ & $2.06 \pm 1.21$ & $3.32 \pm 1.37$ & $1.73 \pm 0.92$ & $0.37 \pm 0.39$ & $0.09 \pm 0.11$ & 2.60 \\
\hline Lepocinclis & - & - & $9.66 \pm 3.87$ & $0.89 \pm 0.84$ & $0.25 \pm 0.46$ & $0.02 \pm 0.05$ & - \\
\hline Phacus & $0.89 \pm 1.01$ & $6.00 \pm 3.47$ & $7.61 \pm 1.94$ & $2.21+1.42$ & $1.50 \pm 0.50$ & $0.29 \pm 0.24$ & 0.28 \\
\hline Trachelomonas & $15.54 \pm .91$ & $12.51 \pm 6.25$ & $18.70 \pm 4.74$ & $40.75 \pm 8.23$ & $8.82 \pm 4.12$ & $6.97 \pm 5.04$ & 3.81 \\
\hline \multicolumn{8}{|l|}{ Chrysophyta } \\
\hline Dinobryon & - & $0.08 \pm 0.19$ & - & - & - & - & - \\
\hline \multicolumn{8}{|l|}{ Dinophyta } \\
\hline Glenodinium & - & - & $0.06 \pm 0.13$ & - & - & - & 0.14 \\
\hline Fungus & $34.60 \pm 9.40$ & $3.60 \pm 2.95$ & $0.72 \pm 0.69$ & $2.94 \pm 2.96$ & $1.10 \pm 0.63$ & $0.28 \pm 0.26$ & - \\
\hline FA & $13.35 \pm 4.25$ & $9.06 \pm 6.80$ & $3.48 \pm 0.86$ & $5.87 \pm 0.63$ & $2.06 \pm 2.70$ & $0.37 \pm 0.32$ & 0.28 \\
\hline Thecamaeba & $4.66 \pm 3.93$ & $5.38 \pm 3.47$ & $0.74 \pm 0.50$ & $5.38 \pm 0.69$ & $6.67 \pm 2.32$ & $0.27 \pm 0.26$ & 0.14 \\
\hline Ciliophora & $1.97 \pm 2.35$ & $0.21 \pm 0.21$ & $0.09 \pm 0.13$ & $2.07 \pm 2.66$ & $0.11 \pm 0.25$ & $0.02 \pm 0.05$ & - \\
\hline
\end{tabular}


Table IV. Continued.

\begin{tabular}{lccccccc}
\hline \multicolumn{1}{c}{ Food items } & L. fuscus & L. podicipinus & B. schneider & P. venulosa & E. ovalis & Hyla nana & *S. fuscomarginatus \\
\hline Rotifera & $5.05 \pm 4.68$ & $1.62 \pm 0.78$ & $2.90 \pm 1.51$ & $13.10 \pm 0.8$ & $5.23 \pm 2.53$ & $0.57 \pm 0.20$ & 0.55 \\
Nematoda & $1.15 \pm 0.36$ & $0.26 \pm 0.38$ & $0.17 \pm 0.26$ & - & $0.22 \pm 0.30$ & - & 0.14 \\
Tardigrada & $0.70 \pm 0.46$ & $0.06 \pm 0.13$ & $0.08 \pm 0.12$ & $0.05 \pm 0.09$ & - & - & - \\
Acari & $0.14 \pm 0.31$ & - & - & - & - & - & - \\
Copepoda & - & - & - & - & $0.06 \pm 0.13$ & - & - \\
Ostracoda & - & - & - & - & $1.80 \pm 3.70$ & - & - \\
Crustacea (UNI) & $3.40 \pm .40$ & $0.44 \pm 0.61$ & $0.36 \pm 0.26$ & $0.28 \pm 0.48$ & $6.12 \pm 5.70$ & $0.14 \pm 0.14$ & 0.48 \\
UNI 1 & - & - & - & $0.05 \pm 0.09$ & $4.64 \pm 2.38$ & $72.54 \pm 6.74$ & - \\
UNI 2 & - & - & - & $0.05 \pm 0.09$ & $0.53 \pm 0.16$ & $8.23 \pm 3.62$ & - \\
\hline
\end{tabular}

Table V. Breadth ( $\mathrm{B}=$ Levins' measure) and diversity ( $\mathrm{H}^{\prime}=$ ShannonWiener index) of the diet of tadpoles in the temporary pond of Nova Itapirema, São Paulo.

\begin{tabular}{lcc}
\hline \multicolumn{1}{c}{ Species } & $\mathrm{B}$ & $\mathrm{H}^{\prime}$ \\
\hline Physalaemus cuvieri & 4.80 & 1.00 \\
Physalaemus centralis & 3.71 & 0.93 \\
Physalaemus nattereri & 2.84 & 0.83 \\
Physalaemus fuscomaculatus & 5.74 & 1.00 \\
Scinax fuscovarius & 5.45 & 1.00 \\
Scinax similis & 4.47 & 0.96 \\
Leptodactylus fuscus & 4.91 & 0.97 \\
Leptodactylus podicipinus & 4.97 & 0.99 \\
Bufo schneider & 5.99 & 1.00 \\
Phrynohyas venulosa & 4.37 & 0.96 \\
Elachistocleis ovalis & 3.70 & 0.93 \\
Hyla nana & 1.80 & 0.58 \\
\hline
\end{tabular}

$1,550 \mu \mathrm{m}$, but at least $52 \%$ of the items were $10-100 \mu \mathrm{m}$ in length (Tab. VII). Niche overlap based on the size of food particles was high $\left(C_{H}>0.54\right)$ for $92.4 \%$ of the 66 possible combinations of species pairs (Tab. VI).

\section{DISCUSSION}

\section{Diet, microhabitat and feeding behavior}

According to ALFORD (1999), relatively little information is available on the diets of tadpoles and field studies of tadpoles diet are not common. Many ingested items pass undamaged through the gut, while other soft-bodied organisms and bacteria are not detected (Hoff et al. 1999). In this study, some of the al gae were found broken and without any organelles in the gut of the tadpoles, but it is not known if the tadpoles digested these algae, because they do not have cellulase for digesting plant materials (Hoff et al. 1999). Euglenoids, along with several species of Chlorophyceae, Dinophyceae and
Baccilariophyceae, were found alive and undamaged in the intestinal contents of Dermatonotus muelleri (Boettger, 1885) and Elachistocleis bicolor (Guérin Méneville, 1838) tadpoles (ECHEVERRíA $\&$ ConfortI 2000). On the other hand, studies have demonstrated that epiphytic diatoms and cyanobacterial endosymbionts enhance growth, development, and survival of Hyla regilla (Baird \& Girard, 1852) (Hylidae) tadpoles (Kupferberg et al. 1994, KUPFERBERG 1997a) and that competition between tadpoles of Rana catesbeiana Shaw, 1802 and R. boylii Baird, 1854 (Ranidae) appeared to be mediated by algal resources (KuPFERBERG 1997b). The detection of Ciliophora and other soft-bodied animals, such as Tardigrada and Rotifera in the intestines, is an important finding because few studies have detected soft-bodied organisms or bacteria, and they could be the real source of nutrients for tadpoles (INGER 1986, Hoff et al. 1999). According Hoff et al. (1999), the contribution of these small items to tadpole diets remains unstudied in nature, and that part of the material that a tadpole ingests that is actually digested is not known.

The unidentified items found in Hyla nana gut contents are similar in shape to members of Bacillariophyceae, but its identification was impossible, because no recognizable structure was found. LAJMANOVICH et al. (2000) suggested that Hyla nana tadpoles have a detritivorous diet, rich in organic matter in decomposition. Thus, these unidentified items may besilica frustule of partially decomposed Bacillariophyceae.

The data obtained suggest the occurrence of food selection, that results in the ingestion of different proportions of the food items available in the habitat rather than in different diet compositions. In choice experiments, KuPFERBERG (1997a) showed that tadpoles foraged selectively on the algal foods that promoted most rapid growth and development.

Duellman \& TRUeB (1986) commented that food partitioning among anuran tadpoles is caused by differences in the ability of the various species to ingest particles of varying sizes and also to the position they occupy in the water column, a consequence of morphological adaptations for the exploitation of specific microhabitats. The high nicheoverlap observed 
Table VI. Niche overlap for the type and size (in bold) of the food items ingested by tadpoles in the temporary pond of Nova Itapirema, São Paulo. For abbreviations see table II.

\begin{tabular}{lccccccccccccc}
\hline & Hn & Pce & Pcu & Pn & Pfm & Lfu & Lp & Sfu & Ssi & Eo & Bs & Pv \\
\hline Hn & - & 0.54 & 0.59 & 0.35 & 0.55 & 0.54 & 0.46 & 0.51 & 0.46 & 0.63 & 0.32 & 0.57 \\
Pce & 0.11 & - & 0.99 & 0.94 & 0.99 & 0.98 & 0.96 & 0.97 & 0.98 & 0.99 & 0.90 & 0.98 \\
Pcu & 0.10 & 0.96 & - & 0.91 & 0.99 & 0.99 & 0.94 & 0.97 & 0.97 & 0.99 & 0.87 & 0.99 \\
Pn & 0.11 & 0.90 & 0.85 & - & 0.90 & 0.88 & 0.99 & 0.86 & 0.91 & 0.92 & 0.99 & 0.86 \\
Pfm & 0.09 & 0.87 & 0.87 & 0.86 & - & 1.00 & 0.92 & 0.98 & 0.98 & 0.98 & 0.85 & 0.99 \\
Lfu & 0.06 & 0.28 & 0.29 & 0.26 & 0.29 & - & 0.90 & 0.99 & 0.98 & 0.97 & 0.83 & 0.99 \\
Lp & 0.11 & 0.94 & 0.93 & 0.85 & 0.82 & 0.47 & - & 0.87 & 0.91 & 0.95 & 0.98 & 0.89 \\
Sfu & 0.07 & 0.67 & 0.66 & 0.70 & 0.86 & 0.25 & 0.60 & - & 0.99 & 0.95 & 0.80 & 0.99 \\
Ssi & 0.06 & 0.45 & 0.43 & 0.52 & 0.69 & 0.23 & 0.40 & 0.93 & - & 0.95 & 0.85 & 0.98 \\
Eo & 0.20 & 0.93 & 0.89 & 0.92 & 0.82 & 0.34 & 0.92 & 0.60 & 0.37 & - & 0.89 & 0.97 \\
Bs & 0.11 & 0.78 & 0.85 & 0.72 & 0.72 & 0.41 & 0.84 & 0.56 & 0.40 & 0.78 & - & 0.81 \\
Pv & 0.10 & 0.33 & 0.37 & 0.29 & 0.35 & 0.52 & 0.50 & 0.31 & 0.35 & 0.40 & 0.64 & - \\
\hline
\end{tabular}

Table VII. Size categories (length in $\mu \mathrm{m}$ ) of the food items consumed and their percent frequencies in the diet of five tadpoles from 13 species. For abbreviations see table II.

\begin{tabular}{cccccccccccccc}
\hline Length of items & Bs & Eo & Hn & Lfu & Lp & Pce & Pcu & Pfm & Pn & Pv & Sfu & Ssi & Sfm \\
\hline $10-50$ & 72.2 & 46.8 & 9.4 & 38.0 & 61.0 & 42.3 & 42.0 & 40.6 & 63.2 & 35.5 & 33.3 & 37.1 & 92.00 \\
$60-100$ & 14.8 & 31.7 & 75.4 & 22.8 & 23.0 & 22.2 & 26.5 & 24.3 & 14.6 & 24.1 & 19.2 & 16.5 & 2.18 \\
$110-150$ & 5.1 & 9.0 & 1.3 & 15.3 & 4.5 & 8.1 & 10.9 & 15.1 & 5.1 & 13.2 & 12.7 & 9.5 & 2.20 \\
$160-200$ & 1.5 & 3.1 & 8.5 & 7.5 & 1.9 & 5.0 & 5.2 & 4.1 & 5.2 & 5.7 & 6.20 & 6.2 & 1.50 \\
$210-250$ & 1.2 & 2.5 & 2.2 & 3.7 & 1.5 & 1.7 & 2.1 & 3.8 & 2.7 & 4.0 & 5.80 & 5.8 & 0.80 \\
$260-300$ & 0.8 & 2.4 & 2.2 & 1.9 & 0.7 & 2.2 & 1.5 & 2.1 & 1.3 & 4.0 & 2.90 & 3.7 & 0.30 \\
$310-350$ & 0.3 & 1.7 & 0.3 & 1.0 & 1.1 & 1.7 & 1.6 & 1.3 & 1.8 & 2.5 & 2.90 & 3.5 & 0.20 \\
$360-400$ & 0.4 & 0.8 & 0.1 & 1.4 & 1.0 & 2.1 & 0.6 & 2.0 & 1.4 & 1.9 & 1.50 & 2.4 & 0.10 \\
$410-450$ & 0.7 & 0.1 & 0.2 & 1.2 & 0.5 & 2.3 & 1.8 & 0.7 & 0.6 & 1.2 & 2.70 & 2.3 & 0.30 \\
$460-500$ & 0.7 & 0.2 & 0.1 & 1.7 & 0.5 & 1.2 & 1.6 & 1.1 & 1.0 & 1.7 & 2.00 & 1.9 & - \\
\hline
\end{tabular}

in the present study demonstrates that particle size is not a criterium due to which food selection occurs. According ALFORD (1999), species differ in their filtration rates and abilities to ingest particles of different sizes, but most are very efficient at extracting a wide variety of particle sizes from the incoming water. In contrast, the influence of the microhabitat on the partitioning of food resources was demonstrated by similarity analysis which divided the tadpoles into two categories: those that mainly ingested diatoms and those that preferred al gae of the genus Oedogonium. The first group contain s both taxonomically related species and species belonging to different families, all of them occurring on the bottom of the pond. The second consisted of two taxonomically related species, Scinax fuscovarius and S. similis, which occurred at the midwater level. Díaz-Paniagua (1985), also detected two clusters in a community of five anuran species in southwestern Spain: bottom tad- poles which mainly ingested detritus and periphytic al gae and midwater tadpoles which mainly ingested phanerogams or phytoplankton. This author proposed that a large proportion of periphytic al gae indicates that the tadpoles are bottom dwellers. In our study area, most diatoms belong to the periphyton community (O. Necchi Jr., pers. comm.), indicating that the tadpoles of the first cluster mainly feed diatoms as a consequence of occupying the same microhabitat.

However, microhabitat partitioning alone does not fully explain the two clusters, because Phrynohyas venulosa tadpoles, although occurring in midwater, presented a different diet compared to the remaining tadpoles that occupied the same microhabitat. Phrynohyas venulosa tadpoles ingested Trachelomonas, a planktonic alga, whereas Scinax fuscovarius and S. similis tadpoles scrape surfaces for food, a fact which explains the large amounts of Oedogonium - a species that grows on leaves of aquatic 
macrophytes and other plants (PRESCOT 1984) - in its diet. Furthermore, Bufo schneideri tadpoles, despite showing a morphology of typical bottom dwellers, were observed swimming near the surface upside down, especially at night. This behavior may explain the preponderance of planktonic algae in their diet. Leptodactylus fuscus tadpoles, despite being typical bottom dwellers (ALTIG \& JOHNSTON 1986), consumed a diet that differed from those of all other tadpoles. These data demonstrate that food partitioning is related not only to the occupation of different microhabitats, but also to the feeding behavior of the tadpoles.

The importance of food partitioning in the organization of tadpolecommunities is controversial. HEYER (1974) and LAJMANOVICH $(1997,2000)$ claimed that space and time were much more important than food partitioning, whereas DiAz-PANIAGUA (1985) distinguished two tadpole clusters on the basis of diet type, microhabitat and morphology. INGER (1986) observed that the different feeding modes of tadpoles are related to microhabitat distribution and to some of the differences in diet composition. In the present study, the low feeding overlap among the species shows that food partitioning was a factor that contributed to tadpole segregation, especially of those belonging to different genera, which occupied different microhabitats and presented different feeding behaviors.

\section{Similarity of diets of related species}

Among tadpoles of Physalaemus Fitzinger, 1826, one species of each taxonomically related pair tended to present greater niche breadth. Thus, P. cuvieri and P. fuscomaculatus had more diversified diet compared with $\mathrm{P}$. centralis and $\mathrm{P}$. nattereri. In contrast, the niche breadth of Scinax fuscovarius and S. similis tadpoles was similar in the variety of items. Niche overlap in food type was higher among P. cuvieri and P. centralis and among $\mathrm{S}$. fuscovarius and $\mathrm{S}$. similis, which presented a lower difference in niche breadth. This suggests that niche overlap may be greater among more generalist species.

In the three pairs of taxonomically related species, niche overlapping was high $\left(C_{H}>0.50\right)$. Except for Physalaemus centralis and P. cuvieri, however, some non-congeneric species (e.g., P. centralis and Leptodactylus podicipinus; P. cuvieri and L. podicipinus) showed equal or higher overlap. These data show that the similarity of the tadpole diet cannot be explained simply by the taxonomic proximity. This does not mean that taxonomic proximity, a historical factor, was not important in the community studied, but contemporary factors, specifically feeding behavior and microhabitat use, also influenced the use pattern of food resources. These data demonstrate that the organization of this community is complex and results from the interaction of several factors.

\section{ACKNOWLEDGMENTS}

We thank Orlando Necchi Jr. and Luís Henrique Branco for training and help with algal identification, and valuable information about algal microhabitat, and Francisco Langeani, all of UNESP, S.J. do Rio Preto, for critical review of the manuscript. Nelson Bernardi and Patricia Toth reviewed English. This study was partially supported by research grants from the Fundação de Amparo à Pesquisa do Estado de São Paulo FAPESP (processes 01/07944-7 and 01/13341-3).

\section{REFERENCES}

Alford, R.A. 1999. Ecology: resource use, competition, and predation, p. 240-278. In: R.W. McDiaRmid \& R. AltIG (Eds). Tadpoles: the biology of anuran Iarvae. Chicago, The University of Chicago Press, XIII+444p.

Altig, R. \& G.F. Johnston. 1986. Major characteristics of freeliving anuran tadpoles. Smithsonian Herpetological Information Service, Washington, (67): 1-75.

ARID, F.M. \& S.F. BARCHA. 1973. Água subterrânea na formação Bauru - Região norte-ocidental do Estado de São Paulo. Boletim de Ciências da Faculdade de Filosofia Ciências e Letras, São José do Rio Preto, 1: 70-101.

Belova, Z.A. 1964. On the feeding of the tadpoles of Rana ridibunda Pall, in the Volga Delta. Zoologiceskij Zurnal, Moscou, 43: 1188-1192.

Bıcudo, C.E.M. \& R.T.M. Bıcudo. 1970. Algas de Águas Continentais Brasileiras: Chave Ilustrada para Identificação de Gêneros. São Paulo, Funbec, 228p.

Diaz-Paniagua, C. 1985. Larval diets related to morphological characters of five anuran species in the Biological Reserve of Doñana (Huelva, Spain). Amphibia-Reptilia, Leiden, 6 : 307-322.

Duellman, W.E. \& L. Trueb. 1986. Biology of Amphibians. New York, McGraw-Hill Book Company, XVII+670p.

Echeverría, D.D. \& V. Confortı. 2000. Euglenoids living in the intestines of microhylid tadpoles of Argentina. Alytes, Paris, 18: 81-89.

GoSNER,.L. 1960. The simplified table for staging anuran larvae with notes on identification. Herpetologica, Lafayette, 16: 183-189.

HEYER, W.R. 1973. Ecological interactions of frog larvae at a seasonal tropical location in Thailand. Journal of Herpetology, Columbus, 7: 337-361.

. 1974. Niche measurements of frog larvae from a seasonal tropical location in Thailand. Ecology, Tempe, 55: 651-656.

. 1976. Studies in larval amphibian habitat partitioning. Smithsonian Contributions to Zoology, Washington, 242: 1-26.

Hoff, K.S.; A.R. Blaustein; R.W. M cDiarmid \& R. Altig. 1999. Behavior: interactions and their consequences, p. 215-239. In: R.W. MCDIARMID \& R. ALTIG (Eds). Tadpoles: the biology of anuran Iarvae. Chicago, The University of Chicago Press, $X I I I+444 p$.

INGER, R.F. 1986. Diet of tadpoles living in a Bornean rain forest. Alytes, Paris, 5: 153-164.

Johnson, L.M. 1991. Growth and development of Iarval 
northern cricket frogs (Acris crepitans) in relation to phytoplankton abundance. Freshwater Biology, Oxford, 25: 51-59.

KREBS, C.J. 1999. Ecological Methodology. Menlo Park, Addison Wesley Longman, $\mathrm{X}+620 \mathrm{p}$.

KUPFERBERG, S.J. 1997a. The role of larval diet in anuran metamorphosis. American Zoologist, Thousand Oaks, 37: 146159.

1997b. Bullfrog (Rana catesbeiana) invasion of a California river: the role of Iarval competition. Ecology, Tempe, 78: 1736-1751.

Kupferberg, S.J.; J.C. M ARKS \& M.E. Power.1994. Effects of variation in natural algal and detrital dietson larval anuran (Hyla regilla) life history traits. Copeia, Lawrence, 1994: 446-457.

LAJMANOVICH, R.C. 1997. Alimentación de larvas de anuros en ambientes temporales del sistema del Río Paraná, Argentina. Doñana Acta Vertebrata, Sevilla, 24: 191-202.

Received in 05.IV.2004; accepted in 03.VIII.2004.
2000. Interpretación ecológica de una comunidad Iarvaria de anfibios anuros. Interciencia, Caracas, 25: 7179.

Lajmanovich, R.C.; M.F. IzaguirRe; M.F. Vera-Candioti \& V.H. Casco. 2000. Uniquestructural pattern of themanicotto glandulare of Hyla nana tadpoles (Anura: Hylidae). AmphibiaReptilia, Leiden, 21: 237-242.

Prescott, G.W. 1984. The Algae: a Review. Koenigstein, Otto Koeltz Science Publishers, 436p.

SeALE, D.B. \& N. BeCKVAR. 1980. The comparative ability of anuran larvae (genera: Hyla, Bufo and Rana) to ingest suspended blue-green algae. Copeia, Lawrence, 1980: 495-503.

WASSERSUG, R.J. 1980. Internal oral features of Iarvae from eight anuran families: functional, systematic, evolutionary and ecological considerations. Miscellaneous Publications Museum of Natural History, University of Kansas, Lawrence, 68: 1-146.

Revista Brasileira de Zoologia 21(4): 745-754, dezembro 2004 